\title{
Distortion of Shape from Motion
}

\author{
Tao Xiang $^{1}$ and Loong-Fah Cheong ${ }^{2}$ \\ ${ }^{1}$ Department of Computer Science \\ Queen Mary, University of London, London E1 4NS, UK \\ txiang@dcs.qmul.ac.uk \\ ${ }^{2}$ Department of Electrical and Computer Engineering \\ National University of Singapore, Singapore 119260 \\ eleclf@nus.edu.sg
}

\begin{abstract}
Given that most current Structure From Motion (SFM) algorithms cannot recover true motion estimates, it is important to understand the impact such motion errors have on the shape reconstruction. In this paper, we present a theoretical model to understand the impact that errors in the motion estimates have on shape recovery. Using this model, we focus on the recovery of second order shape under different generic motions, each of which presenting different degrees of error sensitivity. Understanding such different distortion behaviour is important if we want to design better fusion strategy with other shape cues.
\end{abstract}

\section{Introduction}

In spite of the best efforts of a generation of computer vision researchers, we still do not have a practical and robust system for reconstructing shape from a sequence of moving imagery. Current Structure From Motion (SFM) algorithms perform well only in restricted domains, and different types of algorithms do well on quite different types of sequences. In real life, shape perception typically occurs in a context where a rich nexus of cues are available. If we are able to understand the limits of applicability of the SFM algorithms, in particular the resultant distortion in depth recovered under different motionscene configurations, it then becomes possible to fuse several cues together. Few works have been devoted towards understanding the structural distortion arising from errors in the motion estimates. This paper is a step towards this direction, especially with regards to the recovery of second order curved surfaces from the motion cue.

It is well known that SFM algorithms that recover structure parameters, especially those of second order quantities such as curvatures, are sensitive to various types of errors such as noise $[1,8]$. However, not many researchers have addressed explicitly the problem of reconstruction accuracy given some errors in the 3-D motion estimates. Given that most current structure from motion algorithms cannot recover true motion estimates, our investigation seeks to clarify the impact such motion errors have on the shape estimates; in particular, we attempt to understand the severity of the resultant shape distortion under different motion-scene configurations. Are there generic motion types that can render depth recovery more robust and reliable? What are the likely problem conditions of motion cue? If such understanding could be achieved, we could use other shape cues to 
supplement motion cue when the latter is inadequate so as to achieve robust shape perception.

In this paper, iso-distortion framework [2] is employed to analyze the errors in second order shape recovery, with regards to local representations such as normal curvatures and shape index [5]. As was shown in [3], there exists certain dichotomy between forward and lateral motion in terms of depth recovery. For instance, useful informations like depth order is preserved under a lateral motion in a small field of view, even though it is difficult to disambiguate translation and rotation in such case; while under forward motion, a robust recovery of 3-D structure is much more difficult. In this paper, we make explicit the local shape distortion properties under lateral motion and forward motion.

The main contribution of this paper lies in the elucidation of the impact of errors in 3-D motion estimates on the second order shape recovery in a systematic manner. In particular, our findings show that the second order shape is recovered with varying degrees of uncertainty depending on the types of 3-D motion executed. Furthermore, we also make clear that different shapes exhibit different sensitivities in their recovery to errors in 3-D motion estimates. Evidently, these results remind us the importance of understanding the behaviour of various SFM algorithms under different motion-scene configurations, which in turn might permit a better fusion strategy with other shape cues.

\section{Background}

\subsection{Local shape representation}

To begin, we look at a smooth local surface patch represented by its Taylor series up to the second order terms:

$$
Z=\frac{1}{2}\left(k_{\min } X^{2}+k_{\max } Y^{2}\right)+d+O^{3}(X, Y)
$$

where $k_{\min }, k_{\max }$ are the two principal curvatures with $k_{\min }<k_{\max }$. This represents a canonical case whereby the observer is fixating straight ahead at the surface patch located at a distance $d$ unit away, and the $X$-axis and the $Y$-axis are aligned with principal directions (directions of principal curvatures). Rotating the aforementioned surface patch around the $Z$-axis by $\theta$ in the anti-clockwise direction, we obtain a more general surface patch whose principal directions do not coincide with the $X-Y$ frame:

$$
\begin{aligned}
Z= & \frac{1}{2}\left(\cos ^{2} \theta\left(k_{\min } X^{2}+k_{\max } Y^{2}\right)+\sin ^{2} \theta\left(k_{\max } X^{2}+k_{\min } Y^{2}\right)\right) \\
& +\cos \theta \sin \theta\left(k_{\min }-k_{\text {max }}\right) X Y+d+O^{3}(X, Y)
\end{aligned}
$$

Notice that in either cases the local shape measurements at the fixation point $(\mathrm{X}=\mathrm{Y}=0)$ are good indication of the global second order shape of the surface patch. In the forthcoming analysis, we focus on the second order shape quantities and thus neglect the $O^{3}(X, Y)$ term in Equations (1) and (2).

Local representations of curved surfaces include the classical differential invariants of Gaussian and mean curvatures which are computed based on the principal curvatures. A good shape descriptor should correspond to our intuitive idea of shape: shape is invariant under translation and rotation, and more importantly, independent of the scaling 
operation. Principal curvatures, as well as the Gaussian and mean curvatures satisfy the former but do not satisfy the latter condition because they still contain the information of the amount of curvature. Koenderink [5] proposed two measures of local shape: shape index (S) and curvedness (C) as alternatives to the classical differential shape invariants. $\mathrm{S}$ and $\mathrm{C}$ are defined respectively as follows:

$$
\begin{gathered}
S=\frac{2}{\pi} \arctan \frac{k_{\min }+k_{\max }}{k_{\min }-k_{\max }} \\
C=\sqrt{\frac{k_{\min }^{2}+k_{\max }^{2}}{2}}
\end{gathered}
$$

$S$ is a number in the range of $[-1,+1]$ and obviously scale invariant and $C$ is a positive number with the unit $m^{-1}$. Shape index and curvedness provide us with a description of 3-D quadratic surfaces in terms of their types of shape and amount of curvature.

When shape is reconstructed based on motion cue, it is more appropriate to use shape index as the local measurement of shape type rather than other differential invariants. Due to the well-known ambiguity of SFM, the scale of the recovered objects and the speed of translation can only be determined up to a common factor. The recovered scale information is thus meaningless unless additional information is available. Using shape index, we at least know that it can be estimated correctly when the SFM algorithm gives correct result.

\subsection{General nature of distortion}

This section derives the distortion in the recovered shape given that the motion parameters are imprecise. In the most general case, if the observer is moving rigidly with respect to its 3-D world with a translation $(U, V, W)$ and a rotation $(\alpha, \beta, \gamma)$, the resulting optical flow $(u, v)$ at an image location $(x, y)$ can be expressed as the well-known equation [6]:

$$
\begin{aligned}
u & =u_{\text {trans }}+u_{\text {rot }} \\
& =\left(x-x_{0}\right) \frac{W}{Z}+\frac{\alpha x y}{f}-\beta\left(\frac{x^{2}}{f}+f\right)+\gamma y \\
v & =v_{\text {trans }}+v_{\text {rot }} \\
& =\left(y-y_{0}\right) \frac{W}{Z}+\alpha\left(\frac{y^{2}}{f}+f\right)-\frac{\beta x y}{f}-\gamma x
\end{aligned}
$$

where $\left(x_{0}, y_{0}\right)=\left(f \frac{U}{W}, f \frac{V}{W}\right)$ is the focus of expansion (FOE), $Z$ is the depth of a scene point, $u_{\text {trans }}, v_{\text {trans }}$ are the horizontal and vertical components of the flow due to translation, and $u_{\text {rot }}, v_{\text {rot }}$ the horizontal and vertical components of the flow due to rotation, respectively.

Since the depth can only be derived up to a scale factor, we set $W=1$. Then the scaled depth of a scene point recovered can be written as

$$
Z=\frac{\left(x-x_{0}, y-y_{0}\right) \cdot \mathbf{n}}{\left(u-u_{\text {rot }}, v-v_{\text {rot }}\right) \cdot \mathbf{n}}
$$

where $\mathbf{n}$ is an unit vector which specifies a direction. 
If there are some errors in the estimation of the intrinsic or extrinsic parameters, this will in turn cause errors in the estimation of the scaled depth, and thus a distorted version of the space will be computed. Denoting the estimated parameters with the hat symbol ${ }^{\wedge}{ }^{\wedge}$ ) and errors in the estimated parameters with the subscript $e$ (where the error of any estimate $p$ is defined as $p_{e}=p-\hat{p}$ ), the estimated depth $\hat{Z}$ can be readily shown to be related to the actual depth $Z$ as follows:

$$
\hat{Z}=Z\left(\frac{\left(x-\hat{x}_{0}, y-\hat{y}_{0}\right) \cdot \mathbf{n}}{\left(x-x_{0}, y-y_{0}\right) \cdot \mathbf{n}+\left(u_{\text {rot }_{e}}, v_{\text {rot }_{e}}\right) \cdot \mathbf{n} Z+\left(N_{x}, N_{y}\right) \cdot \mathbf{n} Z}\right)
$$

where $\left(N_{x}, N_{y}\right)$ is a noise term representing the error in the estimate of optical flow. In the forthcoming analysis we emphasize the geometric aspect of the problem and do not attempt to model the statistics of the noise. We will therefore ignore the noise term.

From (6) we can see that $\hat{Z}$ is obtained from $Z$ through multiplication by a factor given by the terms inside the bracket, which we denote by $D$ and call the iso-distortion factor. The expression for $D$ contains the term $\mathbf{n}$ whose value depends on the scheme we use to recover depth. In the forthcoming analysis, we will take this "epipolar reconstruction" approach [2], i.e. $\mathbf{n}$ is along the estimated epipolar direction with $\mathbf{n}=\frac{\left(x-\hat{x_{0}}, y-\hat{y_{0}}\right)}{\sqrt{\left(x-\hat{x}_{0}\right)^{2}+\left(y-\hat{y_{0}}\right)^{2}}}$.

\section{Distortion under lateral motion}

We consider an agent performing a lateral translational motion $(U, V, 0)$, coupled with a rotation $(\alpha, \beta, \gamma)$. It is reasonable to expect that this agent is aware that such a generic type of motion is executed (especially for an active vision system) such that $\hat{W}=W=0$. Under this assumption, the estimated epipolar direction would be a fixed direction for all the feature points with $\mathbf{n}=\frac{(\hat{U}, \hat{V})}{\sqrt{\hat{U}^{2}+\hat{V}^{2}}}$. If the rotation around the optical axis is always not executed (which is usually true for a general visual system) so that the agent sets $\hat{\gamma}=0$, we can further assume $\hat{\gamma}=\gamma=0$. From (6) the distortion factor can be expressed as:

$D=\left(\frac{\left(\hat{U}^{2}+\hat{V}^{2}\right) Z}{(U \hat{U}+V \hat{V}) Z+\hat{U} \beta_{e}\left(X^{2}+Z^{2}\right)-\hat{V} \alpha_{e}\left(Y^{2}+Z^{2}\right)+\left(\hat{V} \beta_{e}-\hat{U} \alpha_{e}\right) X Y}\right)$

from which the mapping from the point $(X, Y, Z)$ to $(\hat{X}, \hat{Y}, \hat{Z})$ can be established. Algebraically, it was shown from [2] that the distortion transformation from the physical to the reconstructed space is of degree two under lateral motion. Thus, a surface of degree $n$ would in general be mapped to one with degree $2 n$. We will concentrate on the second order shape properties of the recovered surface patch.

Consider a surface $\mathbf{s}(X, Y, Z(X, Y))$, parameterized ${ }^{1}$ by $X$ and $Y$, transformed under the mapping to the perceived surface $\hat{\mathbf{s}}(\hat{X}, \hat{Y}, \hat{Z}(X, Y))$. By using Equation (7), the perceived surface can be parameterized by $X$ and $Y$ as follows:

$$
\hat{\mathbf{s}}(X, Y)=(D X, D Y, D Z)
$$

where $D$ is defined in (7) and for brevity, $Z(X, Y)$ has been written as $Z$. Mathematica ${ }^{2}$ is used to investigate the distortion of the local shape in the following section.

\footnotetext{
${ }^{1}$ Such parametric representation may not be possible for more complex global shapes.

${ }^{2}$ Mathematica is a software which can be used for analyzing and visualizing differential geometry problems.
} 


\subsection{Curvatures}

Given this parametric representation of the distorted surface, we can obtain the principal curvatures and principal directions of any point on the distorted surface patch [4]. The expressions of the distorted curvatures under the general case (i.e. any point on the surface patch and any epipolar direction) are very complex. Due to space constraint, we focus on the special case of $\mathbf{n}=(1,0)$ and $(X=Y=0)$, unless otherwise indicated. This case is most relevant to a situated agent, which often executes horizontal lateral motion to judge distance. In such case, it is reasonable to assume that $\hat{V}=0$ although the true $V$ is not necessarily zero due to perturbances. In other words, the estimated epipolar direction can be regarded as along the $X$-axis direction. Furthermore, it is reasonable to believe that the local shape distortion at the fixation point (i.e. $(X=Y=0)$ ) would influence strongly the viewer's perception of the surface in the surrounding region, which can be observed in the simulations and experiments presented later in this paper. For the local surface patch given by Equation (2), we obtain the perceived principal curvatures $\hat{k_{\text {min }}}$ and $\hat{k_{\max }}$ as follows:

$$
\begin{aligned}
& \hat{k_{\text {min }}}=\frac{1}{2}\left(\frac{\left(k_{\min }+k_{\max }\right) U-2 \beta_{e}}{\hat{U}}-\sqrt{\frac{Q}{\hat{U}^{2}}}\right) \\
& \hat{k_{\max }}=\frac{1}{2}\left(\frac{\left(k_{\min }+k_{\max }\right) U-2 \beta_{e}}{\hat{U}}+\sqrt{\frac{Q}{\hat{U}^{2}}}\right)
\end{aligned}
$$

where $Q=U^{2}\left(k_{\min }-k_{\max }\right)^{2}+4\left(\alpha_{e}^{2}+\beta_{e}^{2}\right)-4 U\left(k_{\min }-k_{\max }\right)\left(\beta_{e} \cos 2 \theta+\alpha_{e} \sin 2 \theta\right)$. Notice that the parameter $d$ which indicates the distance between the surface patch and the observer does not affect the distorted principal curvature (and other shape measures based on principal curvatures).

From (9), it is obvious that Gaussian and mean curvatures are not preserved under the distortion; even the signs of principal curvatures are not necessarily preserved. The principal directions of the perceived surface patch can be obtained by computing the eigenvectors of the Weingarten matrix [4] of the parametrical shape representation. It was found that generally the principal directions are not preserved. Only when the principal directions are aligned with the $X-$ and the $Y$-axis and $\alpha_{e}=0$, they are preserved, though the directions of the maximum curvature and that of the minimum curvature may swap.

We are also interested in the distortion in normal curvatures, especially the normal curvatures along the horizontal and vertical directions which are denoted as $Z_{X X}$ and $Z_{Y Y}$ respectively and given by:

$$
\begin{aligned}
Z_{X X} & =k_{\text {min }} \cos ^{2} \theta+k_{\text {max }} \sin ^{2} \theta \\
Z_{Y Y} & =k_{\text {min }} \sin ^{2} \theta+k_{\text {max }} \cos ^{2} \theta
\end{aligned}
$$

The distorted normal curvatures along the horizontal and vertical directions can be obtained as:

$$
\begin{aligned}
\hat{Z}_{\hat{X} \hat{X}} & =\frac{U}{\hat{U}} Z_{X X}-\frac{2 \beta_{e}}{\hat{U}} \\
\hat{Z}_{\hat{Y} \hat{Y}} & =\frac{U}{\hat{U}} Z_{Y Y}
\end{aligned}
$$

Interestingly, $\hat{Z}_{\hat{X} \hat{X}}$ and $\hat{Z}_{\hat{Y} \hat{Y}}$ are not affected by $\alpha_{e}$. Equation (11) shows that normal curvatures along different directions have different distortion properties. 


\subsection{Shape index}

From the expression of the distorted principal curvatures, the distorted shape index at the fixation point can readily be obtained by substituting (9) into (3):

$$
\hat{S}=\frac{2}{\pi} \arctan \left(\frac{\left(k_{\text {min }}+k_{\text {max }}\right) U-2 \beta_{e}}{\hat{U} \sqrt{\frac{Q}{\hat{U}^{2}}}}\right)
$$

where $Q=U^{2}\left(k_{\min }-k_{\max }\right)^{2}+4\left(\alpha_{e}^{2}+\beta_{e}^{2}\right)-4 U\left(k_{\min }-k_{\max }\right)\left(\beta_{e} \cos 2 \theta+\alpha_{e} \sin 2 \theta\right)$.

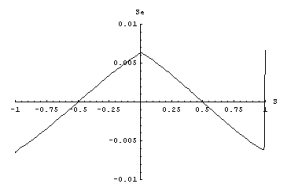

(a)

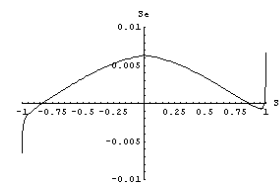

(b)

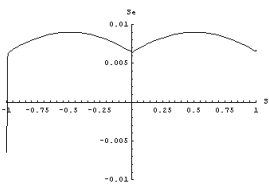

(c)

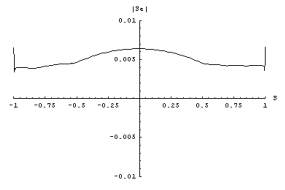

(d)

Figure 1: Distortion of shape index with different principal directions. $\theta=0$ for (a), $\theta=\frac{\pi}{4}$ for (b) and $\theta=\frac{\pi}{2}$ for (c). (d) was obtained by averaging curves with $\theta=0+n \times \frac{\pi}{8}$ where $n=0,1, \cdots 8$. All the other parameters are identical: $\alpha_{e}=0.005, \beta_{e}=-0.02$, $U=0.2$ and $\hat{U}=0.18$.

As the expression for $\hat{S}$ is very complex, we have assorted some diagrams to graphically illustrate the error in the shape index estimate with respect to the true shape index. The curvedness is fixed for each diagram so as to factor out the influence of curvedness. Figure 1 shows the distortion obtained by varying principal directions while fixing all the other parameters. Figures 1(a), (b) and (c) show the sensitivity of shape recovery to the motion estimation errors for three principal directions: $\theta=0, \theta=\frac{\pi}{4}$ and $\theta=\frac{\pi}{2}$. Figure 1(d) show the average sensitivity for different principal directions. Note that the sudden jump of $S_{e}$ in some of the curves corresponds to the case where the direction of the maximum curvature and that of the minimum curvature have swapped. It can also be seen that the principal direction does affect the robustness of the shapes perception. We can also infer from Figure 1 that different shapes have different distortion properties. The average curve shown in Figure 1(d) gives us a rough idea on the overall robustness of the perception of different shapes: the saddle-like shapes (also known as "hyperbolic shapes" with $k_{\min }$ and $k_{\max }$ having different sign) are more sensitive to the errors in 3-D motion estimation than the concave and convex shapes ("elliptic shapes" or "umbilic shapes" with $k_{\min }$ and $k_{\max }$ having same sign).

\section{Distortion under forward motion}

We assume pure forward translation is performed, and the agent executing such motion is aware that such generic type of motion is being executed. We thus have $\hat{U}=U=\hat{V}=$ $V=0$. We also assume $\hat{\gamma}=\gamma=0$ as we did before. Adopting "epipolar reconstruction" approach, the distortion factor can be expressed as:

$$
D=\frac{X^{2}+Y^{2}}{X^{2}+Y^{2}+\alpha_{e} Z\left(X^{2} Y+Y Z^{2}+Y^{3}\right)-\beta_{e} Z\left(X Y^{2}+X Z^{2}+X^{3}\right)}
$$


Following the same procedure of deriving the distorted curvatures as in the lateral motion case, we can obtain the distorted principal and normal curvature expressions on any point of the distorted surface. The distorted shape index and curvedness can also be computed thereafter. These expressions are very complex and are thus not presented here. In particular, at the fixation point, the distorted local shape measurements are undefined due to the undefined distortion factor (see Equation (13)). Our analysis in [3] shows that the distortion factor varies wildly around the fixation point, which implies that the distorted surface patches are also not smooth. Therefore, the distorted local shape measurements at any particular point do not make much sense.

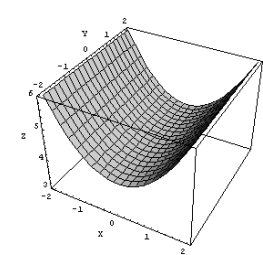

(a)

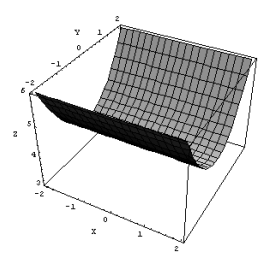

(d)

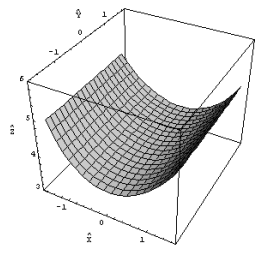

(b)

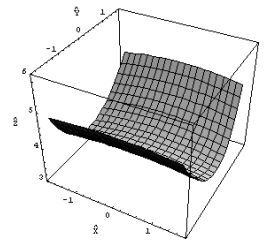

(e)

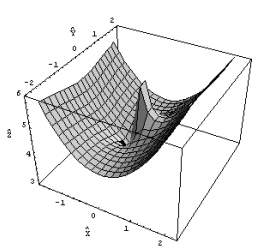

(c)

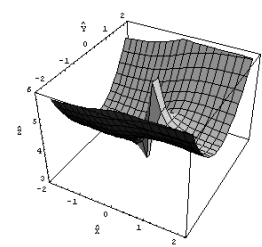

(f)

Figure 2: Distortion of cylinders under lateral and forward motion. (a) and (d) are the original vertical and horizontal cylinders respectively. (b) and (e) are the distorted vertical and horizontal cylinders under lateral motion respectively, with $U=0.9, \hat{U}=1.0$, $V=0.5$ and $\hat{V}=0$. (c) and (f) are the distorted vertical and horizontal cylinders under forward motion respectively. The errors in the estimated rotation are: $\alpha_{e}=0$ and $\beta_{e}=0.05$ for (b) and (e), and $\alpha_{e}=0$ and $\beta_{e}=0.001$ for (c) and (f). The field of view is $127^{\circ}$ for all the diagrams.

To have an idea on how curved surface patches will be distorted under different generic motions, we use some graphical illustrations. We consider a vertical cylinder and a horizontal cylinder, expressed as $Z=\frac{1}{2} X^{2}+4$ and $Z=\frac{1}{2} Y^{2}+4$ respectively. Figure 2 shows the original and recovered surfaces under lateral and forward motions. For the lateral motion case, recall that we have only given the expressions of the estimated curvatures at the fixation point (equations (9) and (11)). Given the parameters in Figure 2, we know that at the fixation point a vertical cylinder will be perceived as a less curved vertical cylinder and a horizontal cylinder will be perceived as a saddle-like shape. The principal directions will not be changed. Figures 2(b) and (e) show that the distorted surface patches are smooth and the distortion behavior at the fixation point seems to be quite representative of the global shape distortion even with a large view angle. The recovered surface patches under forward motion, by contrary, are not smooth and show large distortion. Even when the errors in the rotation estimates are much smaller than those in 
the case of lateral motion, the perceived surface patches are obviously not quadrics with major distortion occurring around the central region of attention.

\section{Experiments}

We conducted experiments based on the SOFA image sequences ${ }^{3}$. SOFA is a package of 9 synthetic sequences designed for testing research works in motion analysis. It includes full ground truth on the motion and camera parameters. Sequence 1 and 5 (henceforth abbreviated as SOFA1 and SOFA5) were chosen for our experiments, the former depicting a lateral motion and the latter a forward motion. Both of them have an image dimension of $256 \times 256$ pixels, a focal length of 309 pixels and a field of view of approximately $45^{\circ}$. The focal length and principal point of the camera were fixed for the whole sequence. Optical flow was obtained using Lucas and Kanade's method [7], with a temporal window of 15 frames. Depth was recovered for frame 9 . We assumed all the intrinsic parameters of the camera were estimated accurately throughout the experiments and concentrated on the impact of errors in the extrinsic parameters.

The 3-D scene for SOFA1 consisted of a cube resting on a cylinder (Figure 3(a)). It is observed that all the feature points on the top face of the cylinder in SOFA1 (which are delineated in Figure 3(a)) lie on a plane. The reconstructed shape of this plane was used to testify our theoretical predictions in the lateral motion case. The camera trajectory for SOFA1 was a circular route on a plane perpendicular to the world $Y$-axis, with constant translational parameter $(U, V, W)=(0.8137,0.5812,0)$ and constant rotational parameters $(\alpha, \beta, \gamma)=(-0.0203,0.0284,0)$. If the observer or the system was aware that a lateral motion is being executed, then $\hat{W}=0$, and the following equation can be used to recover depth:

$$
\hat{Z}=\frac{-f(\hat{U}, \hat{V}) \cdot \mathbf{n}}{(u, v) \cdot \mathbf{n}-\left(\hat{u_{\text {rot }}, v_{\hat{r o t}}}\right) \cdot \mathbf{n}}
$$

The erroneous translation estimates were fixed along the horizontal direction, which means that $\mathbf{n}$ would be fixed as $(1,0)$. The resultant depth recovery when errors in the 3-D motion estimates assumed different values were illustrated in Figure 3 using 3-D plot viewed from the side. In particular, Figure 3(b) shows that the plane (top of the cylinder) remained as a plane, although the noise in the optical flow estimates made some points 'run away' from the plane. Figure 3(c) demonstrates the shape recovered when $\beta_{e}>0$ and $\hat{U}<0$. According to Equation (11), we have $\hat{Z}_{\hat{X} \hat{X}}>0$. It can be seen from Figure 3 (c) that the plane was reconstructed as a convex surface. As expected, when $\beta_{e}<0$ and $\hat{U}<0$, concave surfaces were perceived (Figure 3(d), (e) and (f)). Comparing Figure 3(d) with Figure 3(e) shows that larger $\hat{U}$ resulted in smaller curvature distortion, while comparing Figure 3(d) with Figure 3(f) shows that large $\beta_{e}$ resulted in larger curvature distortion. Figure 3(c)-(f) show that the reconstructed surfaces were not curved in the $Y$ direction. All these results can be explained using Equation (11).

The SOFA5 sequence was used to perform experiments to verify predictions in the case of forward motion. The 3-D scene for SOFA5 comprised of a pile of 4 cylinders stacking upon each other and in front of a frontal-parallel background (Figure 4(a)). The camera trajectory for SOFA 5 was parallel to the world $Z$-axis and the corresponding translational and rotational parameters were $(U, V, W)=(0,0,1)$ and $(\alpha, \beta, \gamma)=(0,0,0)$

\footnotetext{
${ }^{3}$ courtesy of the Computer Vision Group, Heriot-Watt University (http://www.cee.hw.ac.uk/ mtc/sofa)
} 


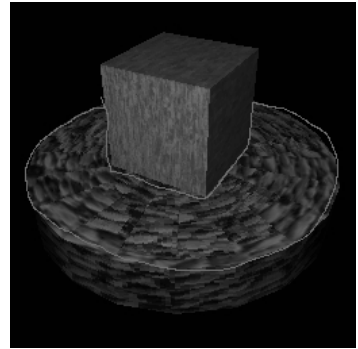

(a)

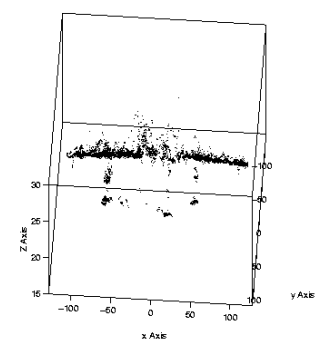

(d)

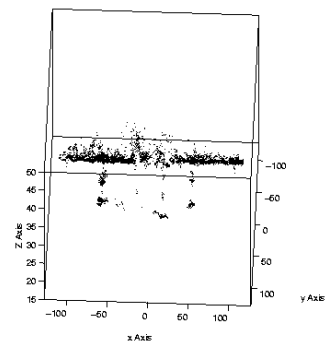

(b)

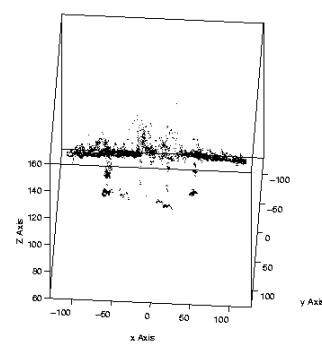

(e)

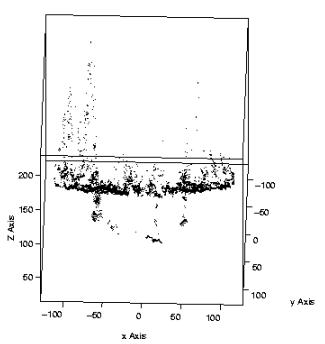

(c)

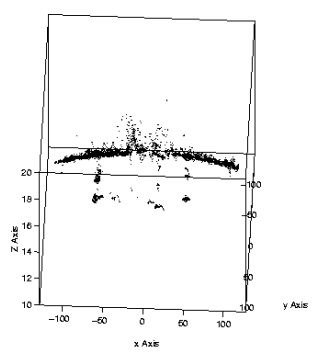

(f)

Figure 3: Lateral motion sequence and depth recovery. (a)SOFA1 frame 9 with the top face of the cylinder delineated; (b) Reconstruction with true motion parameters. (c) Reconstruction with $(U, V=(-1,0)$ and $(\hat{\alpha}, \hat{\beta}, \hat{\gamma})=(-0.03,0.01,0)$. (d) Reconstruction with $(U, V=(-1,0)$ and $(\hat{\alpha}, \hat{\beta}, \hat{\gamma})=(-0.01,0.04,0)$. (e) Reconstruction with $(U, V=(-2,0)$ and $(\hat{\alpha}, \hat{\beta}, \hat{\gamma})=(-0.01,0.04,0)$. (f) Reconstruction with $(U, V=(-1,0)$ and $(\hat{\alpha}, \hat{\beta}, \hat{\gamma})=(-0.01,0.06,0)$.

respectively. We assumed that the observer or the system has been aware that a forward motion is being executed, i.e. $\hat{U}=\hat{V}=0$. Performing epipolar reconstruction, the equation for calculating depth for each feature point would be:

$$
\hat{Z}=\frac{x^{2}+y^{2}}{(u, v) \cdot(x, y)-\left(\hat{u_{\text {rot }}}, v_{\text {rot }}\right) \cdot(x, y)}
$$

Figure 4(b) depicts the case of no errors in the motion parameters. It can be seen that the background plane was preserved roughly. However, when there was small amount of errors in the rotation estimates, a complicated curved surfaced with significant distortion was reconstructed (shown in Figure 4(c)). It is in accordance with our prediction that large depth distortion is expected when forward motion is performed.

\section{Conclusions}

This paper presented an investigation on the reliability of shape recovery from motion cue. Distortion of the local shape recovery was considered under different generic mo- 


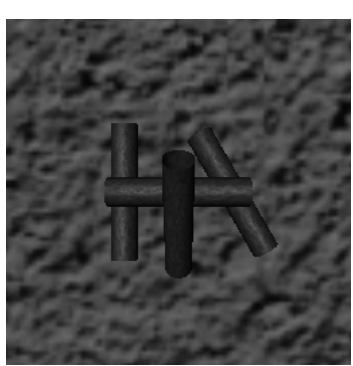

(a)

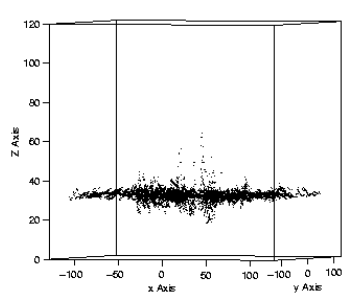

(b)

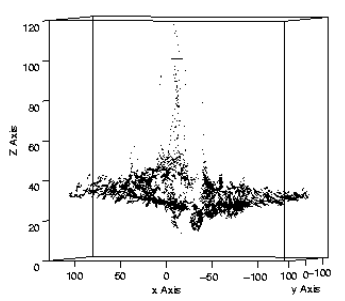

(c)

Figure 4: Forward motion sequence and depth recovery. (a) SOFA5 frame 9. (b) Reconstruction with true motion parameters. (c) Reconstruction with $(\hat{\alpha}, \hat{\beta}, \hat{\gamma})=$ $(-0.001,-0.001,0)$.

tions. Specifically, our distortion model has shown that the types of motions executed are critical for the accuracy of the shape recovered. In the case of lateral motion, the accuracy of curvature estimates exhibits an anisotropy with respect to the estimated translation direction, in that the surface curvature in the estimated translational direction is better perceived than that in the orthogonal direction. Correlatively, the recognition of different shapes (classified in terms of shape index) exhibits different degrees of sensitivity to noise. In the case of forward motion, the reconstructed shape experiences larger distortion compared with the case of lateral motion and is not visually smooth any more.

\section{References}

[1] G. Adiv. Inherent ambiguities in recovering 3-D motion and structure from a noisy flow field. IEEE Trans. PAMI, 11(5):477-489, 1989.

[2] L-F. Cheong and K. Ng. Geometry of distorted visual space and Cremona Transformation. International Journal of Computer Vision, 32(2):195-212, 1999.

[3] L-F. Cheong and T. Xiang. Characterizing depth distortion under different generic motions. International Journal of Computer Vision, 44(3):199-217, 2001.

[4] A. Davies and P. Samuels. An introduction to computational Geometry for curves and surfaces. Clarendon Press, 1996.

[5] J.J. Koenderink and A.J. van Doorn. Surface shape and curvature scales. Image and Vision Computing, 10(8):557-564, 1992.

[6] H.C. Longuet-Higgins. A computer algorithm for reconstruction of a scene from two projections. Nature, 293:133-135, 1981.

[7] B.D. Lucas. Generalized image matching by the method of differences. PhD Dissertation, Carnegie-Mellon University, 1984.

[8] J. Weng, T.S. Huang, and N. Ahuja. Motion and Structure from Image Sequences, Springer-Verlag, 1991. 